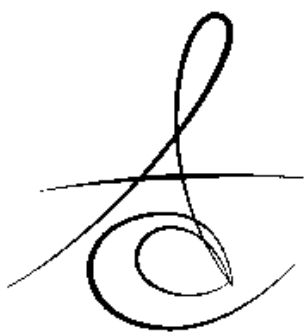

\title{
ESTETİK RESTORATİF CAD/CAM MATERYALLERİN TRANSLÜSENSİ VE RENK STABİLİTESİNİN DEĞERLENDİRİLMESİ $*$
}

\section{EVALUATION OF TRANSLUCENCY AND COLOR STABILITY OF ESTHETIC CAD/CAM RESTORATIVE MATERIALS ${ }^{\neq}$}

\author{
Dr. Öğr. Üyesi Işıl SARIKAYA*
}

Dr. Dt. Ceyda GÜLEÇ**

Makale Kodu/Article code: 3020

Makale Gönderilme tarihi: 19.09 .2016

Kabul Tarihi: 02.12.2016

\section{öz}

Amaç: Estetik restoratif CAD/CAM kompoziti (Lava Ultimate; 3M ESPE, St.Paul, ABD) ve hibrit seramik materyalinin (Vita Enamic, Vita Zahnfabrik, Almanya) translüsensi ve renk stabilitesi hakkında yeterli bilgi bulunmamaktadır. Bu çalışmanın amacı, restorative CAD/CAM materyallerinin translüsensi ve renk stabilitesini araştırmaktır.

Gereç ve Yöntem: Onar örnekten oluşan dört grup, 1 $\mathrm{mm}$ kalınlığında hibrit seramik ve rezin nanoseramik kompozit rezin monolitik bloklardan hazırlandı. Test edilen tüm materyaller için 2M2, yüksek translüsensili bloklar seçildi. Yüzey bitirmelerinde Lava Ultimate örnekler için Sof-Lex (3M ESPE, St.Paul, ABD) diskler, Vita Enamic için Vita Enamic polishing set (Vita Enamic, Vita Zahnfabrik, Almanya) kullanıldı. Örneklerin her iki yüzüne de polisaj uygulandı. Translüsensi parametresi ve renk farklılıkları, örnekler 48 saat kahve solüsyonunda bekletilmeden önce ve sonrasında CIE L*a*b* sisteminde spektrofotometre (Vita Easy Shade, Vita Zahnfabrik, Almanya) ile ölçüldü. Veriler Mann-Whitney U testi ile incelendi $(p<.05)$.

Bulgular: Lava ve Vita Enamic kontrol grupları arasında anlamlı fark görülmedi $(p=1)$. Kahve solüsyonunda bekletilme sonrasında CAD/CAM materyallerin translüsensi parametresi değerleri azalırken $\Delta \mathrm{E}$ renk farklılıklarında artış gözlendi. Kahvede bekletilme sonrasında Lava

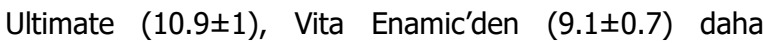
yüksek translüsensi parametresi değeri sergiledi. Ayrıca kahvede bekletilen Lava Ultimate örneklerin $\Delta \mathrm{E}$ değeri $(\Delta \mathrm{E}=3.4)$ Vita Enamic'den $(\Delta \mathrm{E}=1.8)$ daha fazla ve eşik değerin $(\Delta \mathrm{E}=2.7)$ üstünde ölçüldü.

Sonuç: Çalışmamızda Lava Ultimate daha yüksek translüsensi değeri sergiledi ancak daha fazla renk değişimi gösterdi.

Anahtar Kelimeler: Translüsensi; Renk Stabilitesi; CAD/CAM.

\section{ABSTRACT}

Aim: Adequately information is not available about the translucency and color stability of esthetic restorative CAD/CAM composite (LAVA Ultimate, 3M ESPE, StPaul, USA) and hybrid ceramic material (VITA Enamic, Vita Zahnfabrik, Germany). The purpose of this study was to investigate the translucency and color stability of restorative CAD/CAM materials.

Material and Methods: Four groups consist of 10 specimens, were prepared with $1 \mathrm{~mm}$ thickness of hybrid ceramic and resin nano ceramic composite resin monolithic blocks. 2M2 high translucent blocks were chosen for all tested materials. Surface finishing was performed with Sof-Lex (3M ESPE, StPaul, USA) discs for Lava Ultimate specimens and Vita Enamic polishing set (VITA Enamic, Vita Zahnfabrik, Germany) was used for Vita Enamic. The polishing procedures were performed on both sides of the specimens. Translucency parameter and color differences were measured in the CIE L*a*b* system by spectrophotometer (Vita Easy Shade, Vita Zahnfabrik, Germany) before and after 48 hours of immersion in a coffee solution. Data were analyzed with Mann-Whitney $U$ test $(p<.05)$.

Results: There was no significant difference between the Lava control group and Vita Enamic control group $(p=1)$. After immersion of coffee solution, translucency parameter values of CAD/CAM materials were decreased and increased $\Delta \mathrm{E}$ color differences were observed. Significant differences were found among coffee groups for all specimens tested $(p<.001)$. After coffee immersion, Lava Ultimate showed higher translucency parameter value $(10.9 \pm 1)$ than Vita Enamic (9.1 \pm 0.7$)$. Also, $\Delta \mathrm{E}$ values of Lava Ultimate $(\Delta \mathrm{E}=3.4)$ specimens which stored in coffee solution were higher than Vita Enamic $(\Delta \mathrm{E}=1.8)$, and were over than threshold $\Delta \mathrm{E}$ $(\Delta \mathrm{E}=2.7)$

Conclusion: Lava Ultimate showed more translucency parameter value, but also showed more staining in our study.

Keywords: Translucency; Color Stability; CAD/CAM

*Tokat Gaziosmanpaşa Üniversitesi, Diş Hekimliği Fakültesi, Protetik Diş Tedavisi A.D. Tokat.

**Denizli Ağız ve Diş Sağlığı Hastanesi, Denizli. 


\section{Gíriş}

İdeal bir estetik restoratif materyal, doğal diş görünümünü taklit etmelidir. Bu nedenle diş hekimliğinde renk ve materyal seçimi klinik olarak oldukça önemlidir ${ }^{1}$. Restoratif materyallerin rengi ile estetik görünümü birbiriyle yakın ilişkilidir. Ancak renk seçimi, klinik diş hekimliğinde halen en zor karar verilen konulardan biridir. Günümüzde hastaların artan estetik beklentileri de bu seçimi güçleştirmektedir².

Dental seramiklerin mikroyapısının güçlendirilmesi çalışmaları son 20 yıldır artış göstermiştir. Seramiğin optik ve mekanik özelliklerinin güçlendirilmesi için feldspatik porselenin camsı matriksine kristalin yapı ilavesi düşünülmüştür ${ }^{3}$. Alümina ve zirkonya destekli sistemler opak iken lösitle güçlendirilmiş sistemler daha translüsent yapıdadır. Zirkonyum kor yapılı materyallerin opak beyaz rengi ve düşük translüsensi dezavantajının önlenmesi için feldspatik seramiklerin zirkonyum kor üzerine tabakalanması düşünülmüştür. Vita Enamic (Vita Zahnfabrik, Bad Sackingen, Almanya), \%86 (ağırlıkça) seramik içeren polimer infiltre seramik ağ materyali (PICN) olarak tanınan, polimer infiltre feldspatik seramiklerdendir. Seramik ve kompozit materyallerinin ikisinin de özelliklerine sahip olmasının yanında, PICN materyalleri doğal dişi taklit eden mekanik ve estetik olarak benzer karaktere sahiptir ${ }^{4}$.

Dental seramiklerin renk stabilitesi ve yüksek bükülme dayanımı avantajlarına ek olarak, yeni tanıtılan rezin nano seramik (RNC) materyalleri karşı dentisyon için daha az aşındırıcıdır ve minimal diş preparasyonu gerektirmesi ile daha konservatiftir. Lava Ultimate güçlü, aşınmaya dirençli ve yüksek estetik özelliği ile indirekt CAD/CAM restorasyonlar için porselen bloklara alternatif sunmaktadır.

Şüphesiz renk stabilitesi estetik dental restorasyonlarda en önemli klinik faktördür ${ }^{5,6}$. Ayrıca, seramik restorasyonlarının uzun ömürlülüğü ve estetik görünümü renk stabilitesi ve translüsensiye bağlıdır. Translüsensi parametresi (TP) ve kontrast oranı (CR) yaygın olarak dental materyallerin translüsensi belirlenmesi için kullanılmaktadır ve aralarında güçlü bir ilişki bulunmaktadır. TP, siyah ve beyaz arka planda eşit kalınlıktaki materyaller arasındaki renk farkını ifade etmekte$\mathrm{dir}^{8}$. Ancak her malzeme mekanik davranış ve estetik özellikleri bakımından ayrı ayrı değerlendirilmelidir.

CIE L*a*b* renk sistemi; renk algısının gözde 3 farklı renk reseptörüne (kırmızı, yeşil, mavi) dayalı olduğu teorisini desteklemektedir ve halen en popüler renk sistemlerinden biridir ${ }^{9}$. CIE L*a*b* renk sistemi düzenli bir sistemdir, algılanan renk farklılıklarına uygun şekilde eşit aralıklar içermektedir. Bu üç boyutlu renk sisteminde $L^{*}, a^{*}, b^{*}$ eksenleri mevcuttur; L* ekseni, rengin açıklık ve koyuluk koordinatlarını belirler. Saf siyah sıfır $L^{*}$ değerine sahipken, saf beyazın değeri ise 100 'dür. a* değeri kırmızı-yeşil eksenini, b* değeri ise sarı -mavi eksenini oluşturur . Pozitif a ${ }^{*}$ değerleri kırmızıya değişimi gösterirken negatif a *değerleri ise yeşile değişimi gösterir. Benzer şekilde pozitif $b *$ değerleri sarı bileşeni gösterirken negatif $\mathrm{b} *$ değerleri ise mavi bileşenin daha yoğun olduğunu göstermekte$\operatorname{dir}^{9,10}$. CIE L*a*b* sisteminde $\Delta \mathrm{E}$ değeri, iki cisim arasındaki algılanan renk farklılı̆ının miktarını gösteren sayısal bir değerdir. Gruplar arasındaki renk farklılıkları bir formül yardımıyla hesaplanmaktadır ${ }^{8,10}$.

Yeni tanitılan CAD/CAM blokların translüsensi ve renk stabilitesi üzerine literatürde sınırlı veriler bulunmaktadır. Çalışmamızın amacı estetik CAD/CAM materyallerinin translüsensi ve renk stabilitesi özelliklerini karşılaştırmaktır. Çalışmamızın $\mathrm{H}_{\mathrm{o}}$ hipotezi, 'kahve solüsyonu $\mathrm{HC}$ ve RNC CAD/CAM materyallerinde translüsensiyi etkilemez ve renk değişimine neden olmaz' olarak belirlenmiştir.

\section{GEREÇ VE YÖNTEM}

\section{Örneklerin Hazırlanması}

Çalışmada kullanılan HC ve RNC malzemeleri Tablo 1'de listelenmiştir. 1x12x14mm boyutlarındaki örnekler, hassas kesme makinesinde (Micracut 201, Bursa, Türkiye) düşük hızda (150 rpm) su soğutmalı elmas disk ile yüksek translüsent (HT) CAD/CAM bloklardan dilimlenerek elde edilmiştir.

Tablo 1. Çalışmada Kullanılan Seramik Materyalleri

\begin{tabular}{|c|c|c|c|c|c|}
\hline Materyal & Kod & İçerik & $\begin{array}{c}\text { Translüsensi } \\
\text { /Renk }\end{array}$ & Lot no. & Üretici \\
\hline Enamic & $\mathrm{HC}$ & Hibrit Seramik & $\mathrm{HT} / 2 \mathrm{M} 2$ & 51040 & $\begin{array}{c}\text { Vita } \\
\text { Zahnfabrik }\end{array}$ \\
\hline $\begin{array}{c}\text { Lava } \\
\text { Ultimate }\end{array}$ & RNC & $\begin{array}{c}\text { Rezin } \\
\text { Nanoseramik }\end{array}$ & $\mathrm{HT} / \mathrm{A} 2$ & $\begin{array}{c}33140 \mathrm{~A} 2- \\
\mathrm{HT}\end{array}$ & $3 \mathrm{M}$ ESPE \\
\hline
\end{tabular}

Lava Ultimate örnekler için Vitapan klasik renk skalasındaki (Vita Zahnfabrik, Almanya) A2 rengi seçilmiştir. Enamic örnekler için ise Vita 3D renk skalasında 
(Vita Zahnfabrik, Almanya) Vitapan klasik renk skalasında (Vita Zahnfabrik, Almanya) A2 renge karşılık gelen $2 \mathrm{M} 2$ rengi seçilmiştir.

Her iki materyalden ikişer grup $(n=10)$ olmak üzere toplam 40 örnek hazırlanmıştır. Örneklerin her iki yüzeyi de su soğutması altında 100 devir/dk'da 600, 800 ve 1200 grenli silikon karpid zımparalarla (English Abrasives, Londra, İngiltere) zımparalanmıştır. Vita Enamic örneklere Vita Enamic polisaj kiti ile üreticinin tavsiyesi doğrultusunda polisaj yapılmıştır. Lava Ultimate örneklere ise $12.7 \mathrm{~mm}$ çaplı Sof-Lex polisaj diskleri (Sof-Lex polishing discS, 3M ESPE, St.Paul, $A B D$ ) ile üreticilerin önerileri doğrultusunda; kalın ve orta diskler için $10.000 \mathrm{rpm}$, ince ve süper ince diskler için ise $10.000 \mathrm{rpm}$ 'de polisaj uygulanmıştır. Tüm polisaj işlemleri düşük hızlı döner el aletiyle (Kavo Ewl 4990; KaVo Dental Gmbh, Almanya) örneklerin her iki yüzeyine de aynı uygulayıc tarafından yapılmıştır.

Daha sonra tüm örnekler ultrasonik temizleyicide (Pro-Sonic 600; Sultan Healthcare, NJ, ABD) deiyonize su ile 10 sn süreyle temizlenerek sonrasında hava basıncı ile kurulanmıştır. Örnek kalınlıkları dijital kumpas (Absolute Digimatic, Mitutoyo, Japonya) ile ölçülmüştür.

Örnekler her grupta onar adet olmak üzere rastgele seçimle Grup 1; Lava kontrol, Grup 2; Lava kahve, Grup 3; Enamic kontrol ve Grup 4; Enamic kahve olarak 4 gruba ayrılmıştır. Grup 1 ve Grup 3 kontrol grubu olarak belirlenmiş olup örnekler distile suda bekletilmiştir. Grup 2 ve Grup 4'deki örnekler ise kahve solüsyonunda 48 saat bekletilmiştir.

\section{Renk ölçümleri}

Temel renk ölçümleri D65 aydınlatma koşullarında, kahve solüsyonundan önce ve sonra klinik spektrofotometre (Vita Easy Shade Advance, Vita Zahnfabrik, Almanya) ile gerçekleştirilmiştir. CIE L*a*b* sistemine göre renk farklılıklarının $\left(\Delta E^{*}\right)$ tespiti için tüm örnekler beyaz, siyah ve gri zemin üzerinde değerlendirilmiştir ${ }^{8}$. Ölçümler her örnek için 3 kez tekrarlanmış ve ortalaması esas alınmıştır.

CIE L*a*b* sistemine göre $\Delta \mathrm{E}^{*}$ değeri aşağıdaki formülle hesaplanmıştır. Formülde $L_{1} * a_{1} *$, ve $b_{1} *$ ilk ölçüm değerleri, $L_{2} *, a_{2} *$ ve $b_{2} *$ ise ikinci ölçüm değerleridir.

$$
\Delta \mathrm{E}^{*}=\left[\left(\mathrm{L}_{1} *-\mathrm{L}_{2} *\right)^{2}+\left(\mathrm{a}_{1} *-\mathrm{a}_{2} *\right)^{2}+\left(\mathrm{b}_{1} *-\mathrm{b}_{2} *\right)^{2}\right]^{1 / 2}
$$

Translüsensi ise translüsensi parametresi (TP) ile değerlendirilmiştir. Ölçümler D65 standart aydınlatma koşullarında spektrofotometre ile beyaz ve siyah zemin zeminde ölçülmüştür. B siyah zemini ifade ederken $\mathrm{W}$ ise beyaz zemini ifade etmektedir. 100 TP değerindeki bir materyal transparan kabul edilirken 0 TP değeri materyalin opak olduğunu gösterir.

$$
\mathrm{TP}=\left[\left(\mathrm{L}^{*}{ }_{B}-L^{*}{ }_{W}\right)^{2}+\left(a^{*}{ }_{B}-a^{*}{ }_{W}\right)^{2}+\left(b^{*}{ }_{B}-b^{*}{ }_{W}\right)^{2}\right]^{1 / 2}
$$

$\Delta \mathrm{E}^{*}$ tespiti için kullanılan kahve solüsyonu üreticinin önerileri doğrultusunda $300 \mathrm{ml}$ kaynatılmış suya 3.6 g kahve (Nescafe Classic; Nestle, Bursa, Türkiye) eklenerek hazırlanmıştır. Solüsyon 10sn karıştırıldıktan sonra filtre kâğıdından geçirilmiştir. Kahve tüketicilerinin günde 3.2 kupa kahve tükettikleri ve 1 kupa kahveyi içme sürelerinin ortalama $15 \mathrm{dk}$ olduğu düşünüldüğünde çalışmamızda seçilen 48 saatlik bekletilme süresi tüketicilerin 2 aylık kahve tüketimine karşılık gelmektedir ${ }^{11}$. Solüsyon her $8 \pm 1$ saatte bir değiştirilmiştir. Grup 2 ve $4^{\prime}$ deki örnekler 48 saat süreyle $37^{\circ} \mathrm{C}$ ısıda etüvde kahve solüsyonu içinde bekletilmiştir. Daha sonra distile su ile $5 \mathrm{dk}$ süreyle yıkanmış ve renk ölçümleri öncesi kurutma kağıdı (Selpak; Eczacıbaşı, Istanbul, Türkiye) ile kurulanmıştır.

Çalışmamızda translüsent seramiklerde kabul edilen renk değişimi eşik sınırı $\Delta \mathrm{E}^{*}=\mathbf{2 . 7}$ olarak kabul edilmiştir $^{12}$.

\section{BULGULAR}

\section{Renk değişimi}

Verilerin istatistiksel analizi SPSS (SPSS v20.0; IBM SPSS Inc. Chicago, ABD) programı kullanılarak Mann-Whitney $U$ testi $(p<.05)$ ile yapılmıştır. İstatistik sonuçlarına göre Grup 1 ve 3 arasında önemli bir fark bulunmadığı tespit edilmiştir $(p=1)$. Kahve sonrası oluşan renk değişimine göre Grup 2'deki renk değişim farkı $\left(\Delta \mathrm{E}^{*}=3.4\right)$, Grup 4'den yüksek bulunmuştur ki bu değer eşik sınırın $\left(\Delta \mathrm{E}^{*}=2.7\right)$ üzerindedir. Grupların ortalama $\mathrm{L}^{*}, \mathrm{a}^{*}, \mathrm{~b}^{*}$ ve $\Delta \mathrm{E}^{*}$ değerleri ve standart sapmaları Tablo 2 'de listelenmiştir. Grupların $\Delta \mathrm{L}^{*} \Delta \mathrm{a}^{*}$ $\Delta \mathrm{b}^{*}$ karşılaştırması Grafik 1,2 ve 3'de verilmiştir. Ayrıca Şekil 1'de Lava Ultimate ve Vita Enamic örneklerin $\Delta \mathrm{E}^{*}$ değerleri karşılaştırması sunulmuştur.

Tablo 2. Grupların $L^{*}, a^{*}, b^{*}$ değerlerinin ortalama ve standart sapma değerleri.

\begin{tabular}{|c|c|c|c|}
\hline \multirow{2}{*}{ Gruplar } & \multicolumn{3}{|c|}{ Ortalama \pm Standart Sapma } \\
\cline { 2 - 4 } & L* & a* & b* \\
\hline $\mathbf{1}$ & $78.1 \pm 0.4$ & $-3.4 \pm 0.1$ & $9.6 \pm 0.5$ \\
\cline { 2 - 4 } $\mathbf{2}$ & $76.4 \pm 0.5$ & $-3.1 \pm 0.2$ & $12.4 \pm 0.7$ \\
\cline { 2 - 4 } $\mathbf{3}$ & $79.7 \pm 0.4$ & $0.4 \pm 0.1$ & $14.4 \pm 0.5$ \\
\cline { 2 - 4 } & $79.1 \pm 0.4$ & $0.6 \pm 0.1$ & $15.9 \pm 0.4$ \\
\hline
\end{tabular}




\section{Translüsensi Parametresi}

Grup 1 ve Grup 3 arasında önemli derecede fark gözlenmemiştir $(p=1)$. Kahve solüsyonundaki bekletme sonrası Grup 2 ve Grup 4'de TP değerlerinde düşüş gözlenmiştir ve bu gruplar arasındaki farkllık istatistiksel olarak anlamlı bulunmuştur $(p<.001)$. Grup $1-2$ ve grup 3 - 4 arasında istatistiksel olarak önemli farklılık gözlenmiştir $(p<.05)$. Grupların ortalama TP değerleri ve standart sapmaları Tablo 3 'de listelenmi tir. Kahve solüsyonundan sonra Lava Ultimate, $(10,9 \pm 1)$ Vita Enamic'den $(9,1 \pm 0,7)$ daha yüksek TP değeri göstermiştir ve bu durum istatiksel olarak anlamlı bulunmuştur $(p<.05)$.

Tablo 3. Grupların TP değerlerinin ortalama ve standart sapma değerleri.

\begin{tabular}{|l|l|}
\hline Gruplar & $\begin{array}{l}\text { TP (Ortalama } \pm \text { Standart } \\
\text { Sapma) }\end{array}$ \\
\hline 1 & $19.2 \pm 0.6$ \\
\hline 2 & $10.9 \pm 1$ \\
\hline 3 & $19.1 \pm 0.9$ \\
\hline 4 & $9.1 \pm 0.7$ \\
\hline
\end{tabular}

\section{TARTIŞMA}

Çalışmamızın sonuçlarına göre 'kahve solüsyonu hibrit seramik ve rezin nanoseramik CAD/CAM materyallerinde translüsensiyi etkilemez ve renk değişimine neden olmaz' hipotezi reddedilmiştir. Kahve solüsyonunda bekletilme sonrası örneklerin TP değerlerinde azalma ve $\Delta \mathrm{E}$ değerlerinde artış gözlenmiştir. Rezin nanoseramikteki renk değişim miktarı $\left(\Delta \mathrm{E}^{*}=\right.$ 3.4), hibrit seramikten yüksek bulunmuştur ki bu değer eşik kabul edilen sınırın $\left(\Delta E^{*}=2.7\right)$ üzerindedir. Ayrıca translüsensi açısından Lava Ultimate, (10.9 1 ) Vita Enamic'den $(9.1 \pm 0,7)$ daha yüksek TP değeri sergilemiştir.

Johnston ve arkadaşları ${ }^{13}, \Delta \mathrm{E}^{*}=3.7^{\prime}$ nin eşik sınır olarak kabul edildiğini belirtilmiştir ve bu değer yillardır referans olarak belirtilmiştir. Dental seramiklerdeki farklı çalışmalarda $\Delta \mathrm{E}$ değeri 2 ve 4 değerleri arasında değişen referans değerleri alınmıştır ${ }^{5,14-16}$. Ayrıca renk algılama; bireyin renk algısı, materyalin yüzey yapısı, aydınlatıcı koşulları ve renk eşleştirmedeki enstrümantal farkllıkları gibi birçok faktöre bağIıdır $^{17}$. Bu çalışmada, translüsent seramiklerde renk değişimi ortalama eşik değeri için belirtilen $\Delta \mathrm{E}=2.7$ olarak kabul edilmiştir ${ }^{12}$. Günümüzdeki çalışmalarda hala kabul edilen $\Delta \mathrm{E}$ sınır değeri tartışmalı bir konudur ${ }^{17}$.
Acar ve arkadaşları ${ }^{18}$, farklı kalınlıklarda hazırlanan Lava Ultimate ve Vita Enamic'in de aralarında bulunduğu örneklerin kahve içindeki termalsiklus sonrası optik özelliklerini inceledikleri çalışmalarında Lava Ultimate ile nanokompozit rezin olan Filtek Supreme Plus'ın renk değişimini klinik olarak kabul edilemeyen sınırda olduğunu bildirmişlerdir. Ayrıca kahve solüsyonundaki renk stabilitesi açısından minimal invaziv teknikle üretilen $\mathrm{HC}$ materyalinin lityum disilikat seramik bir restorasyona alternatif olabileceği sonucuna varmışlardır ${ }^{18}$.

Materyallerin renklenmesi monomerin hidrofobikliği ve su absorbsiyonu ile ilişkilidir. Bagheri ve arkadaşlarına ${ }^{19}$ göre; kompozitlerdeki su absorbsiyonu, renkli sIVı absorbe ederken oluşan renk değişiminin de göstergesi olacağından önemlidir. RNC; yüksek çapraz bağlı rezin matrikse gömülü nanoseramik partiküller içermektedir ${ }^{20}$. Yaklaşık olarak ağılığının \%80'i nanoseramik parçacıklardan oluşmaktadır ve bu olay nanoteknoloji ve özel ısıl işlemler sonucudur ki bu durum kompozitlerdeki ışık ile polimerizasyon ve otopolimerize durumdan farklıdır ${ }^{21}$.

Çalışmamızda kullanılan HC ise polimer materyal ile doldurulan poröz seramik matriksinden oluşmaktadır. Seramik ağ materyali hidrofobik üretan dimetakrilat (UDMA) ve hidrofilik trietilenglikol dimetakrilat (TEGDMA) karışımı ile infiltre edildiği için ticari olarak hibrit seramik olarak adlandırımışıı ${ }^{22}$. HC ve RNC materyallerin her ikisi de UDMA ve TEGDMA içermektedir ${ }^{23}$. TEGDMA, boyama ajanının rezin matriks içerisine kolay penetre olması özelliği nedeniyle yüksek su emilimine neden olmaktadır ${ }^{24}$. Bu nedenle seramiklerdeki renklenme TEGDMA içeriğine bağı olabilmektedir.

Sonuç olarak her iki HC ve RNC materyali de renklenmeye maruz kalabilmektedir. Ancak bu iki materyalin renk değişimi benzer değildir ve HC ile karşılaşııııldığında, RNC materyali daha fazla renk değişimi göstermektedir. Buna ek olarak, RNC materyali bis-GMA ve onun etoksilenmiş formu olan bisEMA'da içermektedir $^{23}$.

Gajewski ve arkadaşları ${ }^{25}$ ise; UDMA, TEGDMA ve Bis-EMA ile karşılaştıııldığında Bis-GMA'nın yüksek su absorbsiyonuna neden olduğunu bildirmişlerdir. Daha önceki çalışmalarda da UDMA'nın Bis-GMA'dan daha fazla renklenmeye karşı direnci olduğu gösterilmiştir. Bu da UDMA'nın düşük su absorbsiyonu ve çözünürlük karakteri ile ilgilidir ${ }^{26}$. Bu nedenle de Bis- 
GMA monomeri yüksek su absorbsiyonu özelliği nedeniyle RNC materyalindeki renk değişiminden sorumlu tutulmaktadır ${ }^{18}$

Nanodolduruculu partikül boyutlarının yüksek translüsensi göstermesi ise görünür ışığın dalga boyundan daha küçük bir çapa sahip olan parçacıkların daha az ışık saçılması ve emilmesine neden olduğu ile açıklanabilmektedir ${ }^{27}$. Çalışmamızla benzer olarak, Awad ve arkadaşlarının ${ }^{28}$ yaptığı çalışmada $1 \mathrm{~mm}$ ve $2 \mathrm{~mm}^{\prime}$ lik farklı iki kalınlıkta Lava Ultimate, Vita Enamic'e göre daha translüsent bulunmuştur. Lava Ultimate; 500 ve 1200 grenli silikon karbit zımparalarla polisaj ile ve tüm yüzey şartlarında en yüksek TP değeri göstermiştir. Ayrıca Vita Enamic'in (ağırlıkça yaklaşık \%23) yüksek miktardaki $\mathrm{Al}_{2} \mathrm{O}_{3}$ içeriği nedeniyle daha düşük TP değeri gösterdiği sonucuna varılmıştır. Ayrıca materyal içeriği de translüsensiyi önemli şekilde etkilediği bildirilmiştir ${ }^{28}$.

Geleneksel olarak laboratuvarda üretilen indirekt kompozitler ve CAD/CAM rezin içerikli kompozit blokların karşılaştırıldığı bir çalışmada indirekt kompozitlerin renk stabilitesi CAD/CAM rezin bloklardan daha yüksek olarak gözlenmiştir ${ }^{29}$. Ayrıca Pop-Ciutrila ve arkadaşları ${ }^{30}$ Vita Enamic'in, nötr diş rengi ile en iyi uyumlu materyal olduğunu ve bu nedenle ön ve arka grup restorasyonlar için en iyi seçim olacağını bildirmişlerdir.

Gouveia ve arkadaşları ${ }^{31}$ ise matriks monomerleri ve hidrofilite arasındaki ilişkiyi en iyiden kötüye doğru sırasıyla şu şekilde belirtmiştir; TEGDMA, BisGMA, Bis-EMA ve UDMA. Bu monomerler arasında rezin içerisinde $\% 10-15$ oranında bulunan Bis-GMA ve \%10-15 oranındaki TEGDMA sırasıyla \% 3-6 ve \%0-1 su absorbsiyon oranları göstermektedir. $\mathrm{Bu}$ şekilde rezinin önemli derecede bir su absorbsiyon özelliğinin olduğu görülmüştür. Rezin içerisinde bulunan suyun varlığı ise ışık kırılma indeksindeki değişmenin bir sonucu olarak renk değişimlerine neden olmaktadır.

\section{SONUÇ}

Çalışmamızın sınırları dahilinde aşağıdaki sonuçlara ulaşılmıştır:

1. RNC materyali HC'den daha fazla boyanma göstermiştir. Çalışmamızda boyayıcı solüsyon olarak sadece kahve solüsyonu kullanılmıştır. Farklı boyayıcı solüsyonlar ya da termalsiklus ile yaşlandırma ve abrazyon durumu da göz önüne alınarak yeni çalışmalar yapılabilir.

2. Translüsensi açısından kahvede bekletilen RNC materyali $\mathrm{HC}^{\prime}$ den daha yüksek TP değeri sergilemiştir. Çalışmamızın bir diğer sınırlandırması da örneklerin tek bir kalınlıkta hazırlanmış olmasıdır. Farklı kalınlıklarda alınacak örnekler ile translüsensi ve renk değişimi etkileri de ileri çalışmalarda incelenebilir

Işıl Sarıkaya: ORCID ID: 0000-0002-2172-4724

Ceyda Güleç: ORCID ID: 0000-0002-8973-1920

\section{KAYNAKLAR}

1. Barutcigil Ç, Harorlı OT, Yıldız M, Özcan E, Arslan H, Bayındır $F$. The color differences of direct esthetic restorative materials after setting and compared with a shade guide. JADA 2011;142:658-65.

2. Doğan $A$, Yüzügüllü $B$. Renk seçiminde güncel teknolojik gelişmeler [Recent technological developments in color selection]. Atatürk Üniv. Diş Hek. Fak. Derg. 2011;4:65-72.

3. Shillingburg HT, Hobo S, Whitsett LD, Brackett SE. Fundamentals of Fixed Prosthodontics. 3rd ed. Londra; Quintessence Publishing Co Inc:2010. p. 433.

4. Coldea A, Swain MV, Thiel N. Mechanical properties of polymer-infiltrated-ceramic-network materials. Dent Mater 2013;29:419-26.

5. Seghi R, Johnston W, O'Brien W. Performance assessment of colorimetric devices on dental porcelains. J Dent Res 1989;68:1755-9.

6. Heydecke G, Zhang F, Razzoog ME. In vitro color stability of double-layer veneers after accelerated aging. J Prosthet Dent 2001;85:551-7.

7. Barizon KT, Bergeron C, Vargas MA, Qian F, Cobb DS, Gratton DG et al. Ceramic materials for porcelain veneers. Part I: Correlation between translucency parameters and contrast ratio. J Prosthet Dent 2013;110:397-401.

8. Commission Internationale de l'Eclairage (CIE). CIE technical report: colorimetry. [CIE Pub No.15.3]. 2004.

9. Joiner A. Tooth colour: A review of the literature. J Dent, 2004;32:3-12.

10. Powers JM, Sakaguchi RL. Optical, thermal, and electrical properties, In: Craig's Restorative Dental Materials, 12 Ed, Missouri; Mosby:2006. p.28-50.

11. Güler AU, Güler E, Yücel $A C ̧$, Ertaş E. Effects of polishing procedures on color stability of composite 
resins. J Appl Oral Sci. 2009;17:108-12.

12. Ragain JC, Johnston WM. Minimum color differences for discriminating mismatch between composite and tooth color. J Esthet Restor Dent 2001;13:41-8.

13. Johnston W, Kao E. Assessment of appearance match by visual observation and clinical colorimetry. J Dent Res 1989;68:819-22.

14. O'Brien WJ. Dental materials and their selection. 3rd Ed. Chicago; Quintessence Publishing Co Inc: 2008. p. 24-36.

15. Douglas RD, Brewer JD. Acceptability of shade differences in metal ceramic crowns. J Prosthet Dent 1998;79:254-60.

16. Ishikawa-Nagai $S$, Yoshida A, Sakai M, Kristiansen J, Da Silva JD. Clinical evaluation of perceptibility of color differences between natural teeth and allceramic crowns. J Dent 2009;37:57-63.

17. Vichi A, Louca C, Corciolani G, Ferrari M. Color related to ceramic and zirconia restorations: $A$ review. Dent Mater 2011;27:97-108.

18. Acar O, Yılmaz B, Altıntas SH, Chandrasekaran I, Johnston WM. Color stainability of CAD/CAM and nanocomposite resin materials. J Prosthet Dent 2016;115:71-5.

19. Bagheri R, Burrow MF, Tyas M. Influence of foodsimulating solutions and surface finish on susceptibility to staining of aesthetic restorative materials. J Dent 2005;33:389-98.

20. Della Bona A, Corazza PH, Zhang Y. Characterization of a polymer-infiltrated ceramicnetwork material. Dent Mater 2014;30:564-9.

21. Koller M, Arnetzl GV, Holly L, Arnetzl G. Lava ultimate resin nano ceramic for CAD/ CAM: Customization case study. Int J Comput Dent 2012;15:159-64.

22. Mainjot A, Dupont N, Oudkerk J, Dewael T, Sadoun M. From Artisanal to CAD-CAM Blocks State of the Art of Indirect Composites. J Dent Res 2016;95:487-95.

23. Belli R, Geinzer E, Muschweck A, Petschelt A, Lohbauer $U$. Mechanical fatigue degradation of ceramics versus resin composites for dental restorations. Dent Mater 2014;30:424-32.

24. Vasudeva G. Monomer systems for dental composites and their future: a rewiev. J Calif Dent Assoc 2009;37:389-98.
25. Gajewski VE, Pfeifer CS, Froes-Salgado NR, Boaro LC, Braga RR. Monomers used in resin composites: degree of conversion, mechanical properties and water sorption/solubility. Braz Dent J 2012;23:50814.

26. Stawarczyk B, Sener B, Trottmann A, Roos M, Ozcan $\mathrm{M}$, Hammerle $\mathrm{CH}$. Discoloration of manually fabricated resins and industrially fabricated CAD/ CAM blocks versus glass-ceramic: effect of storage media, duration, and subsequent polishing. Dent Mater J 2012;31:377-83.

27. Van Noort R. Introduction to dental materials. 4th Edition: Elsevier Inc. Mosby Ltd; 2013. p. 231-46.

28. Awad D, Stawarczyk B, Liebermann A, Ilie N. Translucency of esthetic dental restorative CAD/CAM materials and composite resins with respect to thickness and surface roughness. J Prosthet Dent 2015;113:534-40.

29. Arocha MA, Basilio J, Llopis J, Bella ED, Roig M, Ardu S, Mayoral JR. Colour stainability of indirect CAD-CAM processed composites vs. conventionally laboratory processed composites after immersion in staining solutions. J Dent 2014;42:831-8.

30. Pop-Ciutrila IS, Dudea D, Eugenia BM, Moldovan M, Cimpean SI, Ghinea R. shade correspondence, color, and translucency differences between human dentine and a CAD/CAM Hybrid ceramic system. J Esthet Restor Dent 2016;28:46-55.

31. Gouveia THN, Publio JC, Ambrosano GMB, Paulillo LA, Aguiar FH, Lim DA. Effect of bleaching on physical properties of a composite resin. Eur J Dent 2016;10:82-91.

\footnotetext{
Yazışma Adresi

Dr. Öğr. Üyesi Işıl SARIKAYA

Tokat Gaziosmanpaşa Üniversitesi,

Diş Hekimliği Fakültesi,

Protetik Diş Tedavisi Anabilim Dalı,

Tokat

TIf: 05425675509

e-mail: sarikayaisil@gmail.com
} 